\title{
Heat conduction of (111) Co/Cu superlattices
}

\author{
Frank Tsui \\ Department of Physics and Astronomy, University of North Carolina, Chapel Hill, \\ North Carolina 27599-3255
}

Baoxing Chen, Joanne Wellman, Ctirad Uher, and Roy Clarke

Department of Physics, University of Michigan, Ann Arbor, Michigan 48109-1120

We report the observation of a large negative magnetothermal resistance in (111) $\mathrm{Co} / \mathrm{Cu}$ superlattices grown by molecular beam epitaxy (MBE) techniques. The observed field dependence is proportional to that of the electrical resistance, in accordance with the Wiedemann-Franz law. The Lorentz number deduced from the measurements is $(2.7 \pm 0.3) \times 10^{-8} \mathrm{~V}^{2} / \mathrm{K}^{2}$. The magnetothermopower also shows a similar correlation with resistivity. These findings reveal that large-angle elastic scattering of conduction electrons, arising from a spin-dependent density of states at the Fermi level, is the dominant process responsible for the observed large magnetotransport effects. In zero field, both electrons and phonons contribute to the thermal conduction of the MBE-grown $\mathrm{Co} / \mathrm{Cu}$ system, at a ratio of about 1:2 near $300 \mathrm{~K}$ becoming nearly equal below $150 \mathrm{~K}$. (C) 1997 American Institute of Physics. [S0021-8979(97)69408-6]

\section{INTRODUCTION}

One of the most exciting discoveries in metallic thin films and multilayers is the observation of giant magnetoresistance (GMR) effects. ${ }^{1-3}$ For obvious scientific and technological reasons, much of the effort has been focused on electrical transport. However, in order to understand the nature of the spin-dependent scattering responsible for the GMR effects, several key transport properties, both electrical and thermal, must be measured. ${ }^{4,5}$ Thermal conductivity measurements can be used to determine the extent of inelastic scattering of the conduction electrons. ${ }^{6}$ When combined with resistivity and thermopower measurements, studies of the thermal conduction provide insight into the mechanism by which conduction electrons scatter, e.g., via spindependent density of states ${ }^{5}$ or by spin-dependent scattering potentials. ${ }^{4}$

The problem in thin film systems is that the large substrate cross section, or specifically the significantly larger substrate thermal conduction compared to that of the film, often makes the desired thermal measurements impractical. Despite this difficulty, thermal properties have been measured in polycrystalline samples of $\mathrm{Co} / \mathrm{Cu}$ multilayers ${ }^{7,8}$ and $\mathrm{Co}-\mathrm{Ag}$ granular alloys, ${ }^{8,9}$ where the requirements on substrates are less stringent than for epitaxial films. In the case of $\mathrm{Co} / \mathrm{Cu}$ samples ${ }^{7,8}$ thin glass slides were used to minimize substrate conduction. In most $\operatorname{cases}^{7-9}$ large-angle elastic scattering was identified as the dominant process for conduction electrons. However, in one set of $\mathrm{Co} / \mathrm{Cu}$ multilayers, ${ }^{8}$ the electron scattering exhibits a significant deviation from the elastic process, a behavior which was attributed to spinflip scattering by long wavelength magnons. ${ }^{8}$

Measurements in epitaxial films are made more difficult by the need to provide a suitable crystalline substrate for oriented growth of the desired structure that is also thin enough not to overwhelm the contribution from the film. A second related difficulty is that the intrinsically slow growth rate, required for maintaining good crystal quality, makes films more than several microns thick impractical. One of the solutions is to find a "removable" substrate. This is the technique discussed in this paper.

Epitaxial films are widely recognized as having wellcontrolled material configurations and properties. Do their much superior crystal coherence lengths and smoother interfaces change the electron scattering processes, particularly spin-dependent scattering? And what is the role of interfaces in these materials? In this paper we address some of these issues, reporting measurements of thermal conduction and other transport properties of (111) $\mathrm{Co} / \mathrm{Cu}$ superlattices grown by molecular beam epitaxy (MBE) techniques. We show that the transport properties of the MBE-grown superlattices obey the Wiedemann-Franz law, so that large-angle elastic scattering, arising from spin-dependent density of states, is the dominant process for conduction electrons giving rise to the large magnetotransport effects. We also demonstrate that the technique employed in this research to measure heat conduction of the epitaxial $\mathrm{Co} / \mathrm{Cu}$ superlattices can be used in other epitaxial systems, particularly fcc (111) and hcp (0001) films.

\section{SAMPLE GROWTH AND CHARACTERIZATION}

The superlattice samples were grown by MBE techniques using synthetic fluorine mica substrates and $\mathrm{Rh}(111)$ buffers. ${ }^{10}$ The growth was carried out in an advanced MBE system equipped with in situ real-time reflection high-energy electron diffraction (RT-RHEED) and a scanning tunneling microscope (STM) to monitor the epitaxial morphology. The mica substrate was subsequently cleaved off the metal film leaving a "free-standing" film which is ideal for thermal transport measurements. The weak bonding of the mica substrates to the metal films allows the post growth cleave-off. Each sample contains more than 200 bilayers of (111) Co and $\mathrm{Cu}$ (of total thickness $\sim 1 \mu \mathrm{m}$ ), grown on a $250 \mathrm{ML}$ thick Rh (111) buffer, and capped with a 10 ML thick Rh layer to protect the superlattice. The Rh buffer was grown at $400{ }^{\circ} \mathrm{C}$ and was subsequently annealed at $800{ }^{\circ} \mathrm{C}$ producing terrace lengths of $\mu \mathrm{m}$ dimensions. The superlattice was deposited at $150{ }^{\circ} \mathrm{C}$ at a rate of $\sim 0.1 \mathrm{ML} / \mathrm{s}$. With these growth 

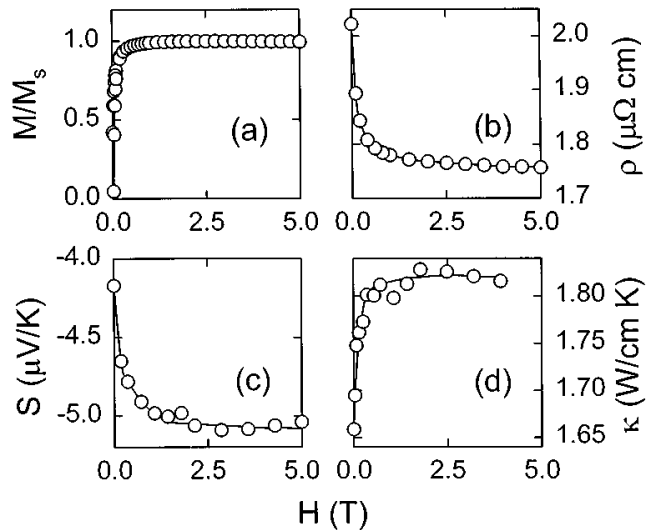

FIG. 1. Field-dependent properties of $\left[\mathrm{Co}_{7 \mathrm{ML}} / \mathrm{Cu}_{19 \mathrm{ML}}\right]_{215}$ at $80 \mathrm{~K}$ : (a) magnetization, (b) resistivity, (c) thermopower, and (d) thermal conductivity. The saturation magnetization in (a), $M_{s}$, is $1400 \mathrm{emu} / \mathrm{cc}$. Lines are to guide the eyes.

parameters the resulting steady-state terrace lengths during growth were on the order of several hundred $\AA$, as measured by RT-RHEED and STM. The system pressure was kept at below $10^{-10}$ Torr during growth.

Thermal transport measurements were performed on strips of free-standing superlattices, typically $1 \mathrm{~mm}$ wide and several mm long. Other transport parameters, including electrical resistance and thermopower, were measured also. The dc 4-terminal technique was used. Magnetotransport measurements were made for fields applied in the growth plane of the superlattices, parallel to the transport current, i.e., longitudinal measurements. A thermal gradient was established by a resistive heater mounted at one end of the film, with the other end anchored on the copper block of the magnet cryostat, and it was measured by constantan-chromel differential thermocouples. Thermopower measurements were performed using calibrated fine $\mathrm{Cu}$ wires. The $\mathrm{Rh}$ contributions to the transport parameters, about $5 \%$ of the total, were measured separately on $\mathrm{Rh}$ films and were subtracted from the data. The magnetization was measured using a commercial SQUID magnetometer.

\section{RESULTS AND DISCUSSION}

The (111) $\mathrm{Co} / \mathrm{Cu}$ superlattices studied here exhibit large longitudinal magnetotransport effects. ${ }^{11,12}$ Figure 1 shows a set of field-dependent measurements, together with the measured magnetization, for $\left[\mathrm{Co}_{7 \mathrm{ML}} / \mathrm{Cu}_{19} \mathrm{ML}\right]_{215}$ at $80 \mathrm{~K}$. In this temperature range both electrical and thermal resistance [Figs. 1(b) and 1(d)] exhibit more than a 10\% change as the field increases to saturation, as does the measured thermopower [Fig. 1(c)]. All longitudinal transport parameters exhibit strong correlation with respect to each other, but they exhibit a much higher saturation field than the measured bulk magnetization [Fig. 1(a)]. The lack of correlation with the magnetization of the Co layers indicates that spin-dependent scattering of conduction electrons occurs not in the bulk of the Co layers but evidently near the $\mathrm{Co} / \mathrm{Cu}$ interfaces. ${ }^{11,12}$

The way the various transport effects scale with each other depends on the nature of the electronic scattering processes. Two correlations are of particular interest for the un-

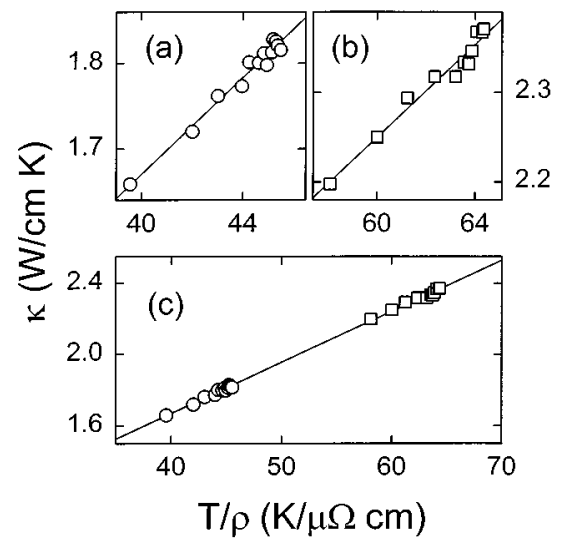

FIG. 2. Scaling plot of magnetothermal conductivity $\kappa(H)$ and magnetoresistivity $\rho(H)$ of $\left[\mathrm{Co}_{7 \mathrm{ML}} / \mathrm{Cu}_{19} \mathrm{ML}\right]_{215}$ at (a) $T=80 \mathrm{~K}$ and (b) $T=150 \mathrm{~K}$. (c) Temperature-dependent scaling plot of $\kappa(H, T)$ vs $T / \rho(H, T)$ at the two temperatures, $80 \mathrm{~K}$ (circles) and $150 \mathrm{~K}$ (squares). The lines through the points are linear fits of the data with slopes that correspond to a Lorentz number of $(2.7 \pm 0.3) \times 10^{-8} \mathrm{~V}^{2} / \mathrm{K}^{2}$.

derstanding of the magnetotransport effects. First, the observed magnetothermal conductivity $\kappa(H)$ satisfies the Wiedemann-Franz law. ${ }^{6}$ This is made evident by the linear behavior of the $\kappa(H)$ vs $T / \rho(H)$ plots shown in Figs. 2(a) and 2(b) for $\left[\mathrm{Co}_{7 \mathrm{ML}} / \mathrm{Cu}_{19 \mathrm{ML}}\right]_{215}$ at two different temperatures, respectively. Furthermore, the temperature dependence also exhibits the same linear behavior, as shown in the $\kappa(H, T)$ vs $T / \rho(H, T)$ plot in Fig. 2(c). From the slope of the linear behavior we obtain a Lorentz number $L_{0}$ of $(2.7 \pm 0.3) \times 10^{-8} \mathrm{~V}^{2} / \mathrm{K}^{2}$. The measured $L_{0}$ is comparable to the nearly-free-electron value of $2.5 \times 10^{-8}$ (Ref. 6). This result indicates that large-angle elastic scattering processes are responsible for the electrical transport effects in MBE-grown (111) $\mathrm{Co} / \mathrm{Cu}$ superlattices. The second correlation is between the thermopower $S$ and resistivity $\rho$. The observed scaling is of the form ${ }^{5,12,13}$

$$
S(H) / T \sim \rho(0) / \rho(H),
$$

where $\rho(0)$ and $\rho(H)$ are resistivities at temperature $T$, at respective fields of 0 and $H$. When scattering of conduction electrons is elastic, scattering by the spin-dependent density of states of the unfilled $d$ bands at the Fermi level leads to precisely this form of scaling. ${ }^{5}$ Therefore, the two observed correlations, one for thermal conductivity and the other for thermopower, together present a clear picture of the scattering mechanism for the conduction electrons responsible for the observed large magnetotransport effects. The lack of spin-dependent scattering at the interior of the Co layers, as indicated by the different saturation behavior between the magnetization and the longitudinal magnetotransport parameters discussed above, highlights the importance of the $\mathrm{Co} / \mathrm{Cu}$ interfacial layers.

We now turn to the temperature dependence of the thermal conductivity. Figure 3 shows the behavior for $\left[\mathrm{Co}_{7 \mathrm{ML}} / \mathrm{Cu}_{19 \mathrm{ML}}\right]_{215}$. At $300 \mathrm{~K}$ the total thermal conductivity $\kappa$ is comparable to that of the bulk $\mathrm{Cu} .{ }^{14}$ It decreases monotonically with temperature, and it becomes smaller than the bulk Co value below $100 \mathrm{~K}$ (Ref. 14). The absence of a low temperature peak, which is present in both bulk $\mathrm{Co}$ and $\mathrm{Cu}$ 


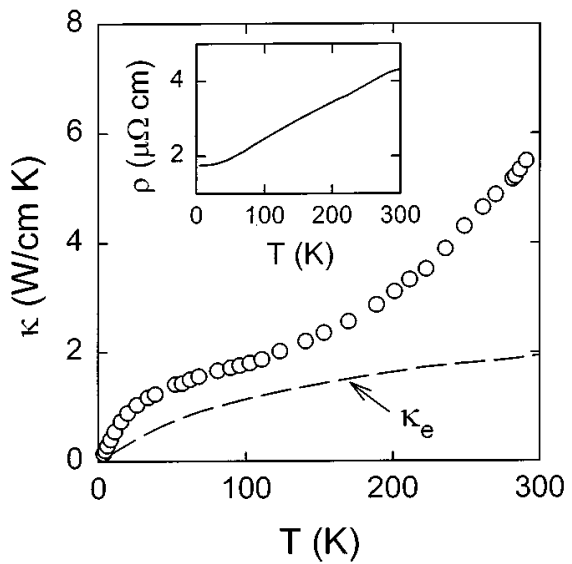

FIG. 3. Temperature-dependent thermal conductivity of $\left[\mathrm{Co}_{7 \mathrm{ML}} / \mathrm{Cu}_{19} \mathrm{ML}\right]_{215}$. Circles are the measured total thermal conductivity. The dashed line indicates the electronic contribution to the thermal conduction $\kappa_{e}$, which is obtained from the resistivity data (inset) using the measured Lorentz number of $2.7 \times 10^{-8} \mathrm{~V}^{2} / \mathrm{K}^{2}$.

crystals, evidently arises from the presence of interfaces. The electronic contribution to the thermal conductivity $\kappa_{e}$ can be estimated from the resistivity data, shown in the inset of Fig. 3, using the measured Lorentz number (see Fig. 3). It contributes about $30 \%$ of the total thermal conduction at 300 $\mathrm{K}$, and the contribution increases to about $60 \%$ at temperatures below $150 \mathrm{~K}$. The reduced electronic contribution in the superlattices also evidently arises from the interfacial scattering. The observed $\kappa_{e}$ is comparable to that of the bulk Co crystals which is much smaller than that of the bulk $\mathrm{Cu}$ (Ref. 14).

We note that the temperature-dependent $\kappa$ vs $T / \rho$ plot, such as the one shown in Fig. 2(c) for $T=80$ and $150 \mathrm{~K}$, does not fall generally on a straight line for the full temperature range of our measurements (between 2 and $300 \mathrm{~K}$ ); only the electronic contribution $\kappa_{e}$ does, because the phonon contribution to heat conduction changes with temperature (Fig. 3).

\section{SUMMARY}

We have studied heat conduction of MBE-grown (111) $\mathrm{Co} / \mathrm{Cu}$ superlattices in a wide range of temperatures and fields using a specially developed "removable substrate" technique. The technique developed here is useful for other epitaxial metallic systems. In this system we observed large magneto-thermal transport effects. Both thermal conductivity and thermopower exhibit scaling with resistivity, and the Wiedemann-Franz law is satisfied. The different contributions from electrons and phonons to the thermal conduction are identified. The results reveal that spin-dependent density of states at the Fermi level plays a dominant role in giving rise to large-angle elastic scattering of the conduction electrons, and leading to the observed large longitudinal magnetotransport effects in this system. The measurements shed light on the basic mechanism of magnetotransport in MBEgrown superlattices, with sharp interfaces, in terms of elastic scattering of the conduction electrons at interfaces.

\section{ACKNOWLEDGMENTS}

We thank D. Barlett and D. Litvinov for assistance. The work was supported in part by ONR Grant No. N00014-92$\mathrm{J}-1335$.

${ }^{1}$ M. N. Baibich, J. M. Broto, A. Fert, F. Nguyen van Dau, F. Petroff, P. Etienne, G. Creuzet, A. Friederich, and J. Chazelas, Phys. Rev. Lett. 61, 2472 (1988).

${ }^{2}$ G. Binasch, P. Grünberg, F. Saurenbach, and W. Zinn, Phys. Rev. B 39, 4828 (1989).

${ }^{3}$ S. S. P. Parkin, R. Bhadra, and K. P. Roche, Phys. Rev. Lett. 66, 2152 (1991).

${ }^{4}$ P. M. Levy, S. Zhang, and A. Fert, Phys. Rev. Lett. 65, 1643 (1990); Phys. Rev. B 45, 8689 (1991)

${ }^{5}$ L. Xing, Y. C. Chang, M. B. Salamon, D. M. Frenkel, J. Shi, and J. P. Lu, Phys. Rev. B 48, 6728 (1993).

${ }^{6}$ J. M. Ziman, Electrons and Phonons (Oxford University Press, Oxford, 1960); R. Berman, Thermal Conduction in Solids (Clarendon, Oxford, 1976).

${ }^{7}$ J. Sakurai, M. Horie, S. Araki, H. Yamamoto, and T. Shinjo, J. Phys. Soc. Jpn. 60, 2522 (1991).

${ }^{8}$ J. Shi, K. Petit, E. Kita, S. S. P. Parkin, R. Nakatani, and M. B. Salamon, Phys. Rev. B 54, (1996).

${ }^{9}$ L. Piraux, M. Cassart, J. Jiang, J. Xiao, and C. L. Chien, Phys. Rev. B 48 , 638 (1993).

${ }^{10}$ F. Tsui, P. D. Han, and C. P. Flynn, Phys. Rev. B 47, 13648 (1993).

${ }^{11}$ D. Barlett, F. Tsui, D. Glick, L. Lauhon, T. Mandrekar, C. Uher, and R. Clarke, Phys. Rev. B 49, 1521 (1994).

${ }^{12}$ F. Tsui, B. Chen, D. Barlett, R. Clarke, and C. Uher, Phys. Rev. Lett. 72, 740 (1994).

${ }^{13}$ J. Shi, R. C. Yu, S. S. P. Parkin, and M. B. Salamon, J. Appl. Phys. 73, 5524 (1993); J. Shi, S. S. P. Parkin, L. Xing, and M. B. Salamon, J. Magn. Magn. Mater. 125, L251 (1993).

14 "Thermal Conductivity-Metallic Elements and Alloys," in Thermophysical Properties of Matter (Plenum, New York, 1970). 